\title{
Anti-D Alloimmunization after RhD-Positive Platelet Transfusion in RhD-Negative Women under 55 Years Diagnosed with Acute Leukemia: Results of a Retrospective Study
}

\author{
Ana Villalba ${ }^{a}$ Marta Santiago $^{a}$ Carmen Freiria $^{a}$ Pau Montesinos $^{a}$ Ines Gomez ${ }^{a}$ \\ Carolina Fuentes $^{b} \quad$ Rebeca Rodriguez-Veiga $^{a} \quad$ José María Fernandez $^{b} \quad$ Guillermo Sanz $^{a}$ \\ Miguel Angel Sanz ${ }^{a}$ Nelly Carpio ${ }^{a}$ Pilar Solves $^{\mathrm{C}}$
}

a Blood Bank, Hematology Service. Hospital Universitari I Politècnic La Fe, Valencia, Spain;

${ }^{b}$ Pediatric Hemato-Oncology Service, Hospital Universitari i Politècnic La Fe, Valencia, Spain;

${ }^{\mathrm{c}}$ CIBERONC, Instituto Carlos III, Madrid, Spain

\section{Keywords}

Platelet transfusion - Anti-D alloimmunization .

Acute leukemia

\section{Summary}

Background: Anti-D alloimmunization can occur when platelets from RhD-positive donors are transfused to RhD-negative patients, due to red blood cell residues in the platelet concentrates. Methods: Our objective was to analyze the anti-D alloimmunization rate in a selected group of women under 55 years of age diagnosed with acute leukemia over an 18-year period. We focused the analysis on RhD-negative patients who received RhDpositive platelet transfusions. Results: From January 1998 to October 2016, 382 women under 55 years were diagnosed with acute leukemia. A total of 56 patients were $\mathrm{RhD}$-negative, and 48 (85.7\%) received $\mathrm{RhD}$-positive platelets. The median number of platelet concentrates transfused per patient was 23 , and $48 \%$ of all platelet transfusions were RhD-positive. The $48 \mathrm{RhD}$ negative patients received a total of 949 RhD-positive platelet concentrates. Two patients developed anti-D: a 36-year-old woman with M3 acute myeloblastic leukemia and a 52-year-old patient with a secondary acute myeloblastic leukemia. Conclusion: We conclude that there is a need for agreement in the transfusion guidelines on the recommendation of anti-D alloimmunization prophylaxis. We suggest a possible benefit in favor of anti-D prophylaxis in childbearing women with acute leukemia.

(c) 2018 S. Karger GmbH, Freiburg

\section{Introduction}

Platelet concentrates (PC) are an essential part of the supportive treatment for patients suffering from malignant hematological diseases [1]. Whenever possible and as outlined in most transfusion guidelines, $\mathrm{PC}$ should match the $\mathrm{ABO}$ and $\mathrm{RhD}$ group [2-4]. However, RhD-negative PC inventories are often not able to fully support $\mathrm{RhD}$-negative patients who require chronic transfusion support. While $\mathrm{ABO}$ antigens are present on the platelet surface, Rh antigens are not. Platelet concentrates are contaminated by varying amounts of red blood cells (RBCs) depending on the technology used to prepare the platelets. Anti-D alloimmunization can therefore occur when platelets from $\mathrm{RhD}$-positive donors are transfused to $\mathrm{RhD}$-negative patients. Various studies of $\mathrm{RhD}$-negative recipients receiving $\mathrm{RhD}$-positive platelet transfusions reported an incidence of $0-19 \% \mathrm{RhD}$ alloimmunization in cancer patients [5-8]. Prevention of anti-D alloimmunization by administering $\mathrm{Rh}$ immunoglobulin is a well-established practice for $\mathrm{RhD}$-negative pregnant women to prevent hemolytic disease of the newborn (HDN). On the contrary, politics regarding immunization prophylaxis in patients receiving $\mathrm{RhD}$-positive $\mathrm{PC}$ is controversial. Some guidelines limit the prophylaxis to women of childbearing age while other institutions do not administer prophylaxis at all $[4,9]$. Our institution does not administer anti-D prophylaxis to patients affected by malignant hematological malignancies, or to women of childbearing age. However, the question remains as to whether this particular group of patients composed of young women could benefit from anti-D prophylaxis.

Our objective was to analyze the anti-D alloimmunization rate in a selected group of women under 55 years of age diagnosed

\section{KARGER}

(c) 2018 S. Karger GmbH, Freiburg

Fax +497614520714 
Table 1. Clinical diagnosis, transfusion history of RhD-positive $\mathrm{PC}$, and alloimmunization in RhD-negative patients $\geq 17$ years

\begin{tabular}{|c|c|c|c|c|c|c|c|}
\hline \multirow[t]{2}{*}{ Diagnosis } & \multirow[t]{2}{*}{ RhD-negative } & \multirow[t]{2}{*}{ RhD-positive } & \multirow[t]{2}{*}{ Median age (range), years } & \multicolumn{3}{|c|}{ Median number of positive PC (range) } & \multirow[t]{2}{*}{ Alloimmunization } \\
\hline & & & & pool & aliquots & apheresis & \\
\hline AML & 18 & $4^{\mathrm{a}}$ & $43(30-55)$ & $12(3-109)^{C}$ & $7.5(4-35)^{\mathrm{e}}$ & $3.5(1-34)^{\mathrm{g}}$ & 1 anti-D + 1 anti-Kell, 1 anti-D \\
\hline ALL & 5 & $2^{\mathrm{b}}$ & $43(39-53)$ & $18(12-33)^{d}$ & $4(1-4)^{f}$ & $2(1-4)^{\mathrm{h}}$ & 0 \\
\hline Total & 23 & 6 & $43(30-55)$ & $14(3-109)$ & $5.5(1-35)$ & $3(1-34)$ & \\
\hline
\end{tabular}

$\mathrm{AML}=$ Acute myelolastic leukemia; $\mathrm{ALL}=$ acute lymphoblastic leukemia; $\mathrm{HSCT}=$ allogeneic hematopoietic stem cell transplantation; $\mathrm{PC}=$ platelet concentrates. ${ }^{\mathrm{a}} \mathrm{HSCT} \mathrm{n}=9$.

${ }^{\mathrm{b}} \mathrm{HSCT} \mathrm{n}=2$.

'Number of patients $\mathrm{n}=16$.

${ }^{\mathrm{d}}$ Number of patients $\mathrm{n}=5$.

${ }^{\mathrm{e} N u m b e r}$ of patients $\mathrm{n}=7$.

fNumber of patients $n=4$.

gNumber of patients $\mathrm{n}=13$.

hNumber of patients $n=5$.

with acute leukemia at our institution in a retrospective way over an 18-year period.

\section{Patients and Methods}

\section{Patients}

We retrospectively reviewed the transfusion data and anti-D alloimmunization rate of women under 55 years of age diagnosed with acute leukemia during the period 1998 to 2016 at la Fe University Hospital. We focused the analysis on RhD-negative patients who received RhD-positive platelet transfusions. Age, underlying diagnosis, $\mathrm{ABO} / \mathrm{Rh}$ type of patient, number and source of $\mathrm{D}$-mismatched platelet transfusions, and date of last antibody screening were recorded. Only patients with a negative antibody screening before RhD-positive platelet transfusion were included.

\section{Transfusion Policy}

Transfusion policy at our center regarding platelet transfusions follows the standards developed by the Spanish Society of Blood Transfusion (SETS) [10] based on international guidelines which recommends the following: i) The threshold for prophylactic transfusion is a pre-transfusion platelet count $\leq 10 \times$ $10^{9} / 1$. If fever $\geq 38,5^{\circ} \mathrm{C}$, sepsis, mucositis $\geq 2$, uncontrolled high pressure, coagulopathy or where acute promyelocytic leukemia is present, the threshold is increased until the pre-transfusion platelet count is $\leq 20 \times 10^{9} / \mathrm{l}$. ii) The threshold for platelet transfusion with the aim of controlling bleeding or increasing the platelet count before an invasive procedure is $<50 \times 10^{9} / 1$. The standard dose for adult patients is $1 \mathrm{PC}$ obtained mostly from whole blood but also from single-donor apheresis. PC were prepared according to the buffy coat method from 4-5 donors per unit and suspended in a platelet-additive solution up to two-thirds of the final volume [11]. Single-donor apheresis platelets were available only in a small quantity. For pediatric patients, an apheresis PC from a single-donor was aliquoted in 4 units and the standard dose was approximately 1 aliquot per $10 \mathrm{~kg}$. Platelet aliquots were transfused to adult patients when expiration was imminent. RBC contamination of platelet preparations was not systematically measured, but only in some units used for quality control. All PC were leukocyte-reduced and irradiated with $25 \mathrm{~Gy}$.

In our institution, $\mathrm{RhD}$-incompatible $\mathrm{PC}$ can be transfused if $\mathrm{RhD}$-compatible products are not available. Anti-D prophylaxis was not administered after $\mathrm{RhD}$-positive $\mathrm{PC}$ transfusion to a $\mathrm{RhD}$-negative recipient.

$\mathrm{ABO}$ detection, Rh typing, and an indirect antiglobulin test (IAT) were done by the automated system ORTHO Autovue Innova (Ortho Clinical Diagnostics, Marlow, UK). Sera of patients with positive tests were tested for specificity using different panels of RBCs (Ortho Clinical Diagnostics; Diamed, Cologne, Germany; and Makropanel 16, Sanqin, Amsterdam, the Netherlands).

\section{Statistics}

Computer software (SPSS, release 13.0; IBM Corporation, Armonk, NY, USA) was used to perform the statistical analysis. Descriptive statistics are presented for variables. Results are expressed as median and range for continuous variables and as numbers with percentages for categorical variables. The Kolmogorov-Smirnov test was employed to investigate the normality distribution of the variables. Categorical variables were compared by means of chi square test or Fisher exact test. The Mann-Whitney U-test for continuous variables was used to compare the groups when applicable. $\mathrm{P}<0.05$ was considered statistically significant.

\section{Results}

From January 1998 to October 2016, 379 women under 55 years of age were diagnosed with acute leukemia: 199 with acute lymphoblastic leukemia (ALL) and 180 with acute myeloblastic leukemia (AML). The median age was 17 years (range $0.11-55$ years). 56 of these patients were RhD-negative, and 48 of them (85.7\%) were transfused with $\mathrm{RhD}$-positive platelets (median age was also 17 years, range $0.5-55$ years). The antibody screen result before receiving $\mathrm{RhD}$-positive platelet transfusion was negative in $46 \mathrm{pa}$ tients, while 2 recipients, both adults and with prior pregnancies, had anti-D: one patient due to a primary anti-D alloimmunization (she was excluded from the study), the other patient received prophylaxis with $\mathrm{D}$ gammaglobulin after a recent birth.

Tables 1 and 2 show the characteristics of RhD-positive platelet transfusions according to the age of patients $(<17$ or $\geq 17$ years). The median number of PC transfused per patient were 23 (range 3-251), and $48 \%$ of all platelet transfusions were RhD-positive. The median RhD-positive PC transfused per patient were 11.5 (range 1-78). The $47 \mathrm{RhD}$-negative patients received a total of 1,023 RhD-positive platelets: 608 platelets from whole blood, 300 aliquots from single-donor apheresis, and 115 apheresis platelets. Patients older than 17 years received significantly more platelet transfusions than younger patients (31.74 vs. $14.0 ; \mathrm{p}=$ 0.029). $66 \%$ of $\mathrm{RhD}$-positive platelets transfused to patients older than 17 years were pooled from whole blood. Patients under 17 years of age received only $30,4 \%$ of pooled platelets, so the ali- 
Table 2. Clinical diagnosis, transfusion history of RhD-positive $\mathrm{PC}$, and alloimmunization in RhD-negative patients $<17$ years

\begin{tabular}{|c|c|c|c|c|c|c|c|}
\hline \multirow[t]{2}{*}{ Diagnosis } & \multirow[t]{2}{*}{ RhD-negative } & \multirow[t]{2}{*}{ RhD-positive } & \multirow[t]{2}{*}{ Median age (range), years } & \multicolumn{3}{|c|}{ Median number of positive PC (range) } & \multirow[t]{2}{*}{ Alloimmunization } \\
\hline & & & & pool & aliquots & apheresis & \\
\hline AML & 4 & $0^{\mathrm{a}}$ & $13(5-15)$ & $6(4-17)^{c}$ & $4.5(2-7)^{\mathrm{e}}$ & $1^{\mathrm{g}}$ & 0 \\
\hline ALL & 20 & $2^{\mathrm{b}}$ & $3(0.5-13)$ & $5(1-30)^{d}$ & $9(2-37)^{\mathrm{f}}$ & $1.5(1-3)^{\mathrm{h}}$ & 0 \\
\hline Total & 24 & 2 & $3(0.5-15)$ & $4(1-30)$ & $7.5(1-37)$ & $1.5(1-3)$ & \\
\hline
\end{tabular}

$\mathrm{AML}=$ Acute myelolastic leukemia; $\mathrm{ALL}=$ acute lymphoblastic leukemia; $\mathrm{HSCT}=$ allogeneic hematopoietic stem cell transplantation; $\mathrm{PC}=$ platelet concentrates. ${ }^{\mathrm{a}} \mathrm{HSCT} \mathrm{n}=0$.

${ }^{\mathrm{b}} \mathrm{HSCT} \mathrm{n}=4$.

'Number of patients $n=3$.

${ }^{\mathrm{d}}$ Number of patients $\mathrm{n}=16$.

eNumber of patients $n=2$.

f Number of patients $n=14$.

gNumber of patients $\mathrm{n}=1$.

${ }^{\mathrm{h}}$ Number of patients $\mathrm{n}=4$.

quoted apheresis platelets were the most frequently transfused. Figure 1 and 2 shows the total RhD-positive platelets transfused and pooled $\mathrm{RhD}$-positive platelets transfused, respectively, according to the age of patients.

16 patients received an allogeneic hematopoietic stem cell transplantation, 9 patients received an $\mathrm{RhD}$-positive graft (median age 39 years, range $0.82-55$ years). None of them developed anti-D.

22 patients (median age 11 years, range $0.5-49$ years) had a serological follow-up of at least 7 days after the last $\mathrm{RhD}$-positive platelet transfusion (median 226 days, range 11-4,955 days). 13 patients were under 17 years of age with a median serological follow-up of 346 days (range 11-4,955 days), the other 9 patients were over 17 years (median serological follow-up 53 days, range 11-459 days). One of these patients (4.5\%), a 36-year-old woman with M3 acute myeloblastic leukemia, who became anti-D alloimmunized after $9 \mathrm{RhD}$-positive platelets unit transfusions (8 platelet pool units, and 1 platelet apheresis unit). Two months later, anti$\mathrm{D}$ was detected. Difference in alloimmunzation rate between pediatric and adult patients was not statistically significant $(\mathrm{p}=0.995)$.

There was a second primary anti-D alloimmunization in the group with a serological follow-up of less than 7 days after the last $\mathrm{RhD}$-positive platelet transfusion. She was a 52 -year-old patient with a secondary acute myeloblastic leukemia evolved from a myelodysplastic syndrome, with a prior transfusion history of $25 \mathrm{RhD}$ positive PC units (24 platelet pool units and 1 platelet aliquot unit). She was also alloimmunized with anti-K. 34 out of the initial 47 patients are still alive (median days of follow-up were 1,710 days, range 18-5,951 days). Of the 2 recipients in whom anti- $\mathrm{D}$ was detected, the patient with AML M3 went into remission and is still alive, but the patient died.

\section{Discussion}

Our study focuses on women under 55 years of age diagnosed with acute leukemia and therefore with high transfusion requirements. To our knowledge, this is the largest study analyzing anti-D alloimmunization after $\mathrm{RhD}$-positive platelet transfusions in $\mathrm{RhD}$ negative females and childbearing women with hematologic malignancy. We have focused our study on this subset of patients (only young women) because of the potential serious consequences of anti-D alloimmunization in future pregnancies (HDN). Previous studies reported an anti-D alloimmunization rate ranging from 0 to $19 \%$ in patients suffering from oncologic diseases and therefore immunosuppressed $[5,9,12-20]$. Lack of anti-D immunization is the most founded result in studies with small numbers of immunosuppressed patients [9, 13, 17-20]. Molnar et al. [21] analyzed 42 pediatric patients receiving $\mathrm{RhD}$-incompatible single-donor platelets and did not find any anti-D alloimmunizations; a study by Cid et al. [18] showed the same results in 22 adult patients diagnosed with different hematologic diseases. These data have led some authors to question whether RhD-positive PC transfusion to RhDnegative recipients should still be a cause of concern [9].

Apart from the immune status of the recipient, another determinant of anti-D alloimmunization is the quantity of residual RBC content in the platelet product, which is the reason of their immunogenic effect. PC can be prepared either by plateletpheresis from single donors or from whole blood units, by pooling 4-6 plateletrich plasma or buffy coat units [23]. In view of the published results, PC prepared from whole-blood have a higher RBC content (range $0.036-0.59 \mathrm{ml}$ ) than PC from single-donor apheresis (range 0.00017-0.009 ml) [22]. It can therefore be deduced that PC from apheresis have a lower risk of producing anti-D alloimmunization. This statement is supported by the literature: in none of the studies in which patients were transfused only with PC from a single-donor apheresis, anti-D alloimmunization was detected [6, 21, 2425 . These data suggest that anti-D immunoprophylaxis is not necessary in $\mathrm{RhD}$-negative patients receiving exclusively $\mathrm{RhD}$-positive apheresis PC. However, there are available data that contradict this: Moncharmont et al. [26] reviewed the alloimmunizations that were performed under the French hemovigilance system: 22 anti-D alloimmunizations were due to $\mathrm{RhD}$-positive platelets from which half were due to single-donor apheresis and half due to pooled platelets. 


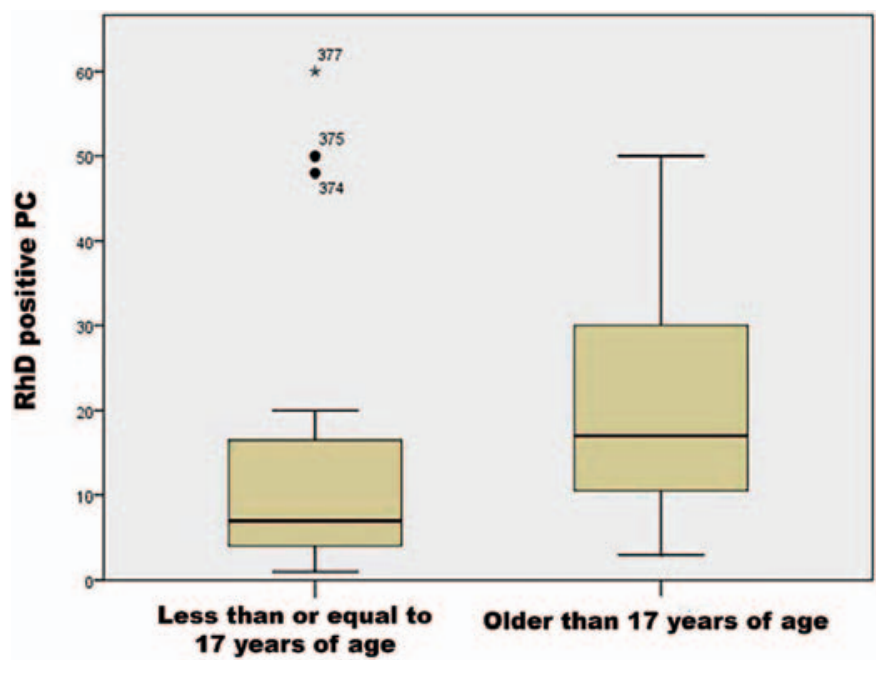

Fig. 1. Boxplot showing the RHD-positive PC transfused to patients according to their age.

Our study has found two cases of anti-D alloimmunization in young women diagnosed with acute leukemia. It has to be highlighted that no child under 17 years of age $(n=23)$ was alloimmunized after RhD-positive platelet transfusions. To our understanding, lack of alloimmunization in the pediatric population could be explained mainly by two reasons: these patients received mostly single-donor apheresis platelets and have less transfusion requirements than adult patients, whereas adult patients received more platelet transfusions, mostly obtained from whole blood by the buffy coat system. Of the two women who become alloimmunized, one had a bad prognosis (secondary leukemia) and died, while the other had a promyelocytic leukemia that has a good prognosis and is currently alive and cured.

The most important reason for administration of prophylactic anti-D gammaglobulin in women of childbearing age is to prevent a future HDN. In fact, anti-D produces the most severe form of immune hemolysis in fetuses and newborns. Therefore, some guidelines such as the American Society of Clinical Oncology or the British Guidelines state that anti-D prophylaxis should be considered for RhD-negative children and for women of childbearing age $[2,4]$. This seems reasonable for immunocompetent patients. However, anti-D administration entails some difficulties in women diagnosed with hematologic malignancies such as acute leukemia, and these should be taken into account: Intramuscular administration is not permitted in severe thrombocytopenic patients as is now the case, the transfusion requirements are high, and they will probably receive frequent $\mathrm{RhD}$-positive platelets for an extended time. The life expectancy can be limited in some patients with the worst prognosis. These may be the reasons why the current guidelines are not strictly followed in hematologic patients and each institution has its own local protocols. Some of these problems can be overcome by intravenous anti-D gammaglobulin administration. Some authors have shown the effectiveness of different intravenous gammaglobulin doses ranging from 100 to 1,000 IU to prevent anti-D development after RhD-positive PC transfusion [16, 24].

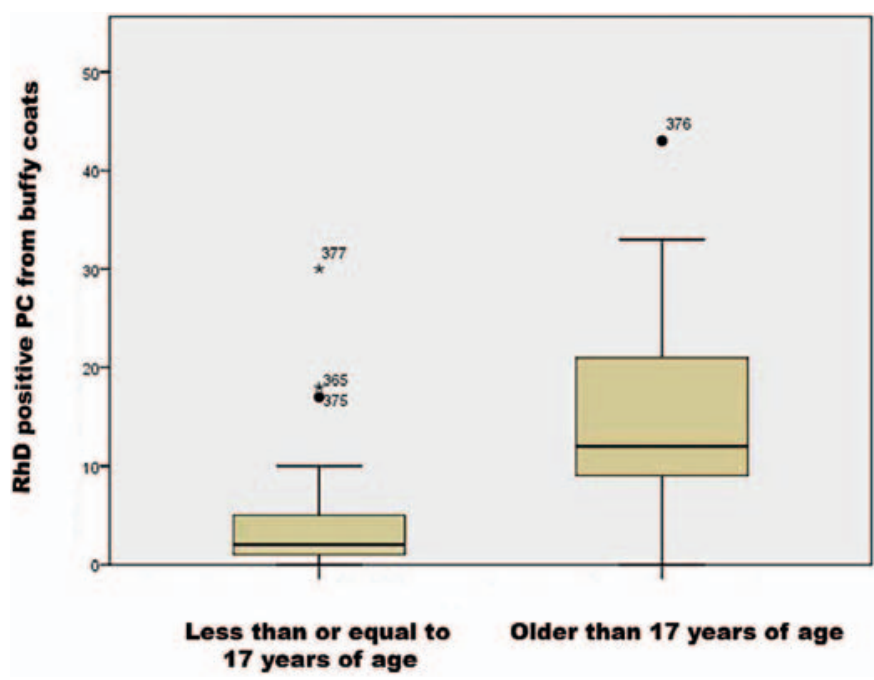

Fig. 2. Boxplot showing the pooled RhD-positive PC transfused to patients according to their age.

In conclusion, alloimmune response to $\mathrm{RhD}$-positive PC from whole blood can occur in patients diagnosed with acute leukemia. This is the reason why we conclude the need for agreement in the transfusion guidelines on the recommendation of anti-D alloimmunization prophylaxis in this type of patients. Although the number of patients in our study is too small to reach a conclusion, we suggest a possible benefit in favor of anti-D prophylaxis in childbearing women with acute leukemia and good prognosis, e.g. promyelocytic leukemia, taking into account the serious complications derived from an anti-D alloimmunization in patients of fertile age who can overcome the disease and want a pregnancy. On the other hand, we cannot suggest any recommendation regarding pediatric prophylaxis, although the data may suggest a lower risk of alloimmunization than in adults, as has also been described in previous studies. Nevertheless, more studies would be needed to provide sufficient data to plan any revision of the current guidelines.

\section{Authors' Contributions}

Pilar Solves analyzed data and wrote the paper; Ana Villalba designed the study and collected data; Carmen Freiría, Marta Santiago, and Rebeca Rodriguez-Veiga collected data; Inés Gómez, Pau Montesinos, and Carolina Fuentes collected data; Guillermo Sanz, José María Fernández, Miguel Ángel Sanz, and Nelly Carpio analyzed data.

\section{Funding Sources}

No funding.

\section{Disclosure Statement}

Authors declare no conflict of interests. 


\section{References}

1 Cid J, Harm SK, Yazer MH: Platelet transfusion. The art and science of compromise. Transfus Med Hemother 2013;40:160-171.

2 Schiffer CA, Anderson KC, Bennett CL, et al: Platelet transfusion for patients with cancer: clinical practice guidelines of the American Society of Clinical Oncology. J Clin Oncol 2001;19:1519-1538.

3 Liumbruno G, Bennardello F, lattanzio A, et al: Recommendations for the transfusion of plasma and platelets. Blood Transfus 2009;7:132-150.

4 Escourt LJ, Birchall J, Allard S, Bassey SJ, Hersey P, Kerr JP, et al: Guidelines for the use of platelet transfusions. Br J Haematol 2016;176:365-394.

5 Cid J, Lozano M, Ziman A, West KA, O’Brien KL, Murphy MF, Wendel S, Vazquez A, Ortín X, Hervig TA, Delaney M, Flegel WA, Yazer MH: Low frequency o anti-D alloimmunization following $\mathrm{D}+$ platelet transfusion: the anti-D alloimmunization alter D-incompatible platelet transfusions (ADAPT) study. $\mathrm{Br} \mathrm{J}$ Haematol 2015;168:598-603.

6 O’Brien JL, Haspel RL, Uhl L: Anti-D alloimmunization alter D-incompatible platelet transfusions: a 14year single-institution retrospective review. Transfusion 2014;54:650-654.

7 Shaz BH, Hillyer CD: Residual risk of D alloimmunization: is it time to feel safe about platelets from $\mathrm{D}+\mathrm{do}-$ nors?. Transfusion 2011;51:1132-1135.

8 Cid J, Carbassé G, Pereira A, Sanz C, Mazzara R, Escolar G, Lozano M: Platelet transfusions from D+ donors to D-patients: a 10-year follow-up study of 1014 patients. Transfusion 2011;51:1163-1169.
Bartley AN, Carpenter JB, Berg MP: D+ platelet transfusions in D- patients: cause of concern? Immunohematology 2009;25:5-8.

10 Sociedad Española de Transfusión sanguínea y terapia Celular: Guía sobre la transfusión de componentes sanguíneos y derivados plasmaticos. Barcelona, SETS, 2010

11 Larsson S, Sandgren P, Sjodin A, et al: Automated preparation of platelet concentrates from pooled buffy coats: in vitro studies and experiences with Orbisac system. Transfusion 2005;45:743-751.

12 Goldfinger D, McGinniss MH: Rh incompatible platelet transfusions. Risks and consequences of sensitizing immunosuppressed patients. N Engl J Med 1971,284: 942-944.

13 Lichtiger B, Surgeon J, Rhorer S: Rh-incompatible transfusion therapy in cancer patients. Vox Sang 1983; 45:139-143.

14 Baldwin ML, Ness PM, Scott D, Braine H, Kickler TS: Alloimmunization to D antigen and HLA in D-negative immunosuppressed oncology patients. Transfusion 1988;28:330-333.

15 McLeod BC, Piehl MR, Sassetti RJ: Alloimmunization to $\mathrm{RhD}$ by platelet transfusions in autologous bone marrow transplant recipients. Vox Sang 1990;59:185-189.

16 Heim MU, Bock M, Kolb HJ, et al: Intravenous anti-D gammaglobulin for the prevention of rhesus isoimmunization caused by platelet transfusions in patients with malignant diseases. Vox Sang 1992;62:165-168.

17 Atoyebi W, Mundy N, Croxton T, Littlewood TJ, Murphy MF: Is it necessary to administer anti-D to prevent $\mathrm{RhD}$ immunization alter the transfusion of RhD-positive platelet concentrates? Br J Haematol 2000;111: 980-983.
8 Cid J, Ortin X, Elies E, Castella D, Panades M, MartínVega C: Absence of anti-D alloimmunization in hematologic patients alter D-incompatible platelet transfusions. Transfusion 2002;42:173-176.

19 Misso S, Feola B, Paesano L, et al: Anti-D alloimmunisation following Rh-incompatible platelet transfusions. Blood Transfus 2006;4:102-105.

20 Solves P, Carpio N, Gomez I, et al: Risk of Rh(D) alloimmunisation after $\mathrm{Rh}(\mathrm{D})$ positive platelet transfusions in patients undergoing hematopoietic stem cell transplantation. Transfus Med 2015;25:49-50.

21 Molnar R, Johnson R, Sweat LT, Geiger TL: Absence of alloimmunization in $\mathrm{D}$ - pediatric oncology patients receiving D-incompatible single-donor platelets. Transfusion 2002;42:177-182.

22 Cid J, Yazer MH, Lozano M: Platelet transfusion and respecting patient D type. Curr Opin Hematol 2015;22: 540-546.

23 Hellstern P: Efficacy and adverse events of platelet transfusion-product-specific differences. Transfus Med Hemother 2008;35:102-105.

24 Zeiler T, Wittmann G, Zingsem J, et al: A dose of 100 IU intravenous anti-D gammaglobulin is effective for the prevention of $\mathrm{RhD}$ immunization after $\mathrm{RhD}$-incompatible single donor platelet transfusion. Vox Sang 1994;66:980-983.

25 Weinstein R, Simard A, Ferschke J, et al: Prospective surveillance of $\mathrm{D}$ - recipients of $\mathrm{D}+$ apheresis platelets: alloimmunisation against $\mathrm{D}$ is not detected. Transfusion 2015;55:1327-1330.

26 Moncharmont P, Barday G, Meyer F: Red blood cell alloimmunisation after platelet transfusion: a 5-year study. Blood Transfus 2014;12:147-148. 